\title{
Langues perdues, langues retrouvées : le témoignage de Cathy
}

Gabriella Vernetto

\section{OpenEdition}

1 Journals

Édition électronique

URL : http://journals.openedition.org/esp/3731

DOI : 10.4000/esp.3731

ISSN : 2532-0319

Éditeur

Centre d'Information sur l'Éducation Bilingue et Plurilingue

Édition imprimée

Date de publication : 1 juin 2019

Pagination : 19-24

ISSN : 1127-266X

\section{Référence électronique}

Gabriella Vernetto, «Langues perdues, langues retrouvées : le témoignage de Cathy », Éducation et sociétés plurilingues [En ligne], 46 | 2019, mis en ligne le 01 février 2021, consulté le 01 mars 2021. URL : http://journals.openedition.org/esp/3731 ; DOI : https://doi.org/10.4000/esp.3731

Ce document a été généré automatiquement le 1 mars 2021.

(c) CIEBP 


\title{
Langues perdues, langues retrouvées : le témoignage de Cathy
}

\author{
Gabriella Vernetto
}

\author{
L'auteure tient à remercier Mme Cathy \\ Castiglioni d'avoir accepté de participer à la \\ rédaction de cet article. Le texte est la synthèse \\ d'un questionnaire d'enquête, d'une interview et \\ de parties tirées du Portfolio de l'étudiant du \\ cours de Didactique du plurilinguisme, a.a. \\ 2017/2018, de l'Université de la Vallée d'Aoste.
}

1 Née en Belgique, Cathy a toujours vécu en Vallée d'Aoste. Sa mère est belge et son père italien, ils se sont rencontrés en Italie et, après une période de vie commune en Belgique, ils ont décidé d'aménager en Vallée d'Aoste. Cathy étudie à l'Université de la Vallée d'Aoste, à la faculté de sciences de la formation pour les professeurs des écoles. En plus de son activité d'étudiante, elle effectue des remplacements dans les écoles maternelles et élémentaires de la région.

Elle a fait sa scolarité entièrement en Vallée d'Aoste: l'école de l'enfance, primaire et secondaire de premier degré à Cervinia, à la montagne, et l'école secondaire de second degré ${ }^{1}$ en ville, à Aoste. Cervinia est une station de ski renommée et ses écoles accueillent beaucoup d'enfants étrangers (suédois, bulgares, américains, albanais,...) dont les parents travaillent sur les pistes de skis ou dans l'hôtellerie et la restauration.

En famille, son papa et son grand-frère parlent italien, sa mère flamand. La mère est bilingue (français - flamand), mais elle a décidé de parler flamand à ses enfants parce qu'elle a estimé qu'ils apprendraient le français à l'école. En plus, Cathy a été exposée au français en contexte familial parce que sa grand-mère maternelle est francophone.

4 À l'école, elle a appris le français, qui est enseigné en Vallée d'Aoste à parité horaire avec l'italien depuis la maternelle, et a été sensibilisée à l'anglais, toujours à la maternelle, grâce à un cours donné par la maman d'une de ses copines, d'origine américaine. Sa langue forte, celle qu'elle utilise le plus souvent, reste l'italien, sauf 
quand elle fait des séjours en Belgique, où le flamand devient la langue principale de communication en alternance avec le français et l'italien.

Elle n'a jamais appris le flamand en contexte scolaire. Elle possède, donc, une excellente compétence à l'oral, comprend sans difficultés des textes écrits, mais, comme elle a appris à le lire et l'écrire toute seule, elle avoue avoir encore du mal avec son orthographe.

Enfin, elle a des compétences réceptives en piémontais, en francoprovençal et en allemand, langues qu'elle entend dans son environnement social en Vallée d'Aoste.

Dans son Portfolio du cours de Didactique du plurilinguisme, elle décrit de la manière suivante les langues qu'elle connaît. Les étudiants devaient colorier une silhouette en attribuant à chaque langue qu'ils connaissaient une couleur différente et rédiger par la suite un texte pour justifier leurs choix ${ }^{2}$.

«J'ai colorié de cette façon ma silhouette: dans la tête les langues étrangères (français et anglais) que je connais et que j'emploie à l'école pour des tâches qui requièrent raisonnement et concentration. Pour la même raison, l'italien aussi occupe une partie de la tête car c'est la langue dans laquelle je pense le plus souvent. Les oreilles représentent les langues que je comprends mais que je ne parle pas (piémontais, francoprovençal et allemand). Les bras et les pieds sont coloriés avec les langues (italien, anglais, français) qui me permettent de voyager et de connaître d'autres personnes. Une bonne partie du corps est flamand car cela représente mes racines comme aussi les langues qui sont dans mon cœur car je les connais depuis que je suis petite. Enfin il y a une partie que j'ai laissée blanche parce qu'il y a de la place pour d'autres langues que je voudrais apprendre à l'avenir"

8 Toujours dans son Portfolio, elle livre une réflexion sur le regard que les autres portent sur son identité multiple :

«Ce qui m'a toujours gênée par rapport à comment les autres me voient, c'est qu'ils pensent que je suis ou belge ou italienne. Je m'explique: en Italie, plutôt qu'italienne je suis considérée comme belge, même si j'ai toujours vécu ici, (...) quand je suis en Belgique, c'est l'inverse. Mais moi, je sens que mon identité n'est ni l'une ni l'autre, elle est le mélange des deux. Je ne peux pas les séparer car l'une a toujours grandi avec l'autre. Elles communiquent, se renforcent réciproquement, en générant ce que je suis "

9 Au cours de son interview, elle souligne l'importance de maîtriser deux langues et deux cultures, malgré l'ambiguïté que cela comporte, à l'aide d'une anecdote. Quand elle était petite, elle passait ses vacances à la mer, en Belgique, où elle servait d'interprète entre les enfants francophones et flamands. Seulement dans ces occasions, quand elle était présente, les enfants des deux communautés parvenaient-ils à jouer tous ensemble. Mais juste pour lui faire plaisir et parce qu'ils la considéraient comme l'« Italienne ».

\section{Devenir plurilingue, rester plurilingue}

Cathy a appris le français pendant ses vacances en Belgique, c'est sa grand-mère maternelle qui lui parlait cette langue. Quand elle a commencé à apprendre le français à l'école, elle a pourtant refusé de l'utiliser pour s'exprimer. La compréhension ne posait aucun problème, mais elle n'arrivait pas à parler spontanément comme quand elle était en famille. Ce n'est qu'à l'âge de 8 ans, à l'occasion des vacances d'été en Belgique, qu'elle a pris goût à l'expression orale et depuis lors, elle n'a plus eu de 
problème, à l'école non plus. Elle a retrouvé tout le bagage linguistique qu'elle avait stocké au cours de années précédentes et elle a commencé à l'utiliser pour produire des phrases, des discours, des textes.

Le français et le flamand restent cependant ses langues faibles, celles qu'elle utilise le moins. Avec son père elle parle italien, ainsi qu'avec son frère, même s'ils s'amusent à faire des jeux de mots en flamand. En principe, elle parle flamand avec sa mère, mais des fois elle lui répond en italien. Sa vie sociale se passe en Vallée d'Aoste où les occasions de parler le français, et encore moins le flamand, sont rares.

Son espace culturel aussi (livres, films, spectacles,...) est principalement italophone, même si elle est capable de lire et de comprendre parfaitement des ouvrages dans les autres langues. Elle se sent profondément flamande, comme elle l'a déclaré dans son Portfolio, à tel point que quand sa mère parle en néerlandais au téléphone ou par skype avec des clients des Pays-Bas, tout en comprenant ce qu'elle dit, elle ressent une espèce de gêne, parce qu'elle n'entend pas les mêmes sonorités que lorsqu'elle parle en flamand.

Elle se rend compte qu'elle est en train de perdre ses langues et ce sentiment l'inquiète. Pendant le cours de didactique du plurilinguisme les étudiants avaient le droit de s'exprimer dans la langue dans laquelle ils se sentaient le plus à l'aise. Cathy s'est toujours exprimée en français et dans son Portfolio elle explique ce choix :

«Moi, pendant toutes mes interventions j'ai essayé de parler le plus possible en français parce que, par rapport à quand j'étudiais au lycée, je n'ai plus eu beaucoup d'occasions de m'exprimer dans cette langue. Comme avec le flamand je me rends compte que je suis en train de perdre les langues que j'emploie le moins et c'est dommage!»

Dans son interview, elle évoque également un sentiment d'insécurité vis-à-vis de ces deux langues. Le français, langue apprise à l'oral en famille et puis à l'école, dont elle a une excellente maitrise, est ressenti comme une langue fragilisée, elle ne se sent pas sûre de ce qu'elle dit quand elle l'utilise pour s'exprimer. Pour le flamand, c'est plutôt l'écrit qui pose problème. Elle a appris à lire et surtout à écrire toute seule et des fois elle n'est pas sûre de l'orthographe des mots. Cependant, dans son Portfolio, elle souligne la fierté d'avoir su accomplir cet exploit :

«Parmi les souvenirs les plus clairs que j'ai de mon enfance il y a les moments passés à lire des bandes dessinées en flamand. Je me rappelle encore bien le jour où, dans le grenier de ma grand-mère j'ai trouvé des vieux albums de Tintin. Je les ai tout de suite feuilletés car j'aimais tellement les dessins animés de Tintin qu'en découvrant que les histoires étaient tirées de ces albums j'en ai été ravie. En sachant déjà décoder les graphèmes, j'ai commencé à les lire, à comprendre les mots écrits en y associant après les phonèmes corrects. J'avais appris à lire en flamand toute seule, quelle joie! Depuis ce jour-là je dévorais une bande dessinée après l'autre pendant mes vacances en Belgique, jusqu'à un jour où j'ai lu aussi, avec pas mal de difficultés, un livre! »

Si on la met en situation d'activité de classe, où elle est l'experte de la langue, tout se passe sans problème. L'exercice était le suivant: à l'aide de quatre phrases en flamand, accompagnées de la traduction en français, les étudiants devaient trouver la traduction d'une quatrième phrase du français vers le flamand (Armand, Maralliet 2013).

«En connaissant le flamand je n'ai pas pu faire cet exercice mais observer mes camarades le faire et voir ensuite leur phrase a été très amusant pour moi. En plus, en étant l'experte en ce moment j'ai aussi eu l'occasion d'expliquer la solution et d'en trouver une autre en corrigeant légèrement une phrase qui avait été traduite 
presque parfaitement. En effet la première solution possible de la phrase 'Hier, nous avons vu un chat' est Gisteren, hebben wij een kat gezien. Mais si on ne fait pas l'inversion sujet-verbe la phrase peut aussi être traduite comme Wij hebben gisteren een kat gezien »

\section{Le rôle de l'école dans l'ouverture aux langues et aux cultures}

Son statut de plurilingue et de future enseignante lui permet de réfléchir au rôle que l'école pourrait jouer dans la sensibilisation à la diversité linguistique et culturelle, notamment dans une perspective d'accueil et d'intégration des élèves allophones. Voici son témoignage sur l'accueil d'une petite Suédoise en classe de primaire :

«En habitant Cervinia, quand j'étais petite j'ai eu l'occasion de connaître des enfants venant d'autres pays comme le Brésil, la Bulgarie et la Suède mais je n'ai aucun souvenir d'activités liées à l'interculturalité. Je me rappelle très bien le jour où une fille suédoise est arrivée dans notre classe: on était tous très intéressés, car c'était la nouveauté et on l'a bien acceptée, même si elle ne parlait pas un mot d'italien. Aujourd'hui, je me demande si la situation aurait été pareille avec un enfant marocain, car avec le petit bulgare et le brésilien on n'a pas été également accueillant »

Et plus loin, toujours dans son Portfolio, elle réfléchit sur le rôle que l'école pourrait avoir vis-à-vis des élèves allophones, de la reconnaissance des langues familiales et de l'accueil des parents (Vernetto 2017) :

«J'imagine que des parents immigrés, qui peut-être parlent peu ou pas du tout l'italien, se sentent accueillis et plus à l'aise, quand ils rentrent à l'école pour accompagner leur enfant, s'ils y trouvent une classe remplie de panneaux écrits en plusieurs langues dans laquelle ils retrouvent aussi leur langue maternelle. Cela montre une ouverture de l'école et des enseignants envers eux et permet d'établir plus facilement une relation de confiance et de respect qui est à la base de l'alliance éducative. Si les parents sont sereins quand ils accompagnent leur enfant, c'est plus facile que l'élève aussi soit plus à l'aise à l'école »

\section{BIBLIOGRAPHIE}

ARMAND F., E. MARAILLET. 2013. Éducation interculturelle et diversité linguistique, ELODIL - en ligne http://www.elodil.umontreal.ca/guides/education-interculturelle-et-diversite-linguistique/

DERYCKE M. 2014. Le suivi des enfants en voyage et l'usage du portfolio, quelques résultats, Éducation et Sociétés Plurilingues $n^{\circ} 36$ (juin): 25-38.

Portfolio Européen des langues - pour les élèves de 9 à 11 ans. 2004. Modèle accrédité n 65/2004. Autonome Provinz Bozen Südtirol, Région autonome Vallée d'Aoste - Assessorat de l'Éducation et de la Culture. http://www.scuole.vda.it/images/adattamenti/primaria.zip 
VERNETTO G. 2017. Quand les parents ouvrent plus grand les portes des langues familiales à l'école. Les témoignages des conteurs, p. 114-128 in L. REVELLI, A. TABOURET-KELLER, G. VARRO, Langues faibles - Lingue deboli, L'Harmattan Italia.

VeRnETTO G. 2005. L'expérimentation du Portfolio en Vallée d'Aoste, Éducation et Sociétés

Plurilingues $\mathrm{n}^{\circ} 19$ (décembre): 21-26.

\section{NOTES}

1. Nous utilisons la dénomination officielle en vigueur en Vallée d'Aoste pour indiquer les différents cycles scolaires. L'école de l'enfance (3 ans de scolarité) accueille les enfants âgés de 3 à 5 ans, l'école primaire ( 5 ans de scolarité) va de 6 à 10 ans, l'école secondaire de premier degré (3 ans de scolarité) de 11 à 13 ans, l'école secondaire de second degré ( 5 ans de scolarito) de 14 à 18 ans.

2. L'activité est tirée du Portfolio Européen des Langues pour le primaire (2004) de la Vallée d'Aoste et du Sud-Tyrol (Vernetto 2005). Sur l'importance de l'utilisation du Portfolio, même avec des enfants en bas âge, voir DERYCKE M. 2014. Le suivi des enfants en voyage et l'usage du portfolio, quelques résultats, Éducation et Sociétés Plurilingues n 36 (juin): 25-38.

\section{RÉSUMÉS}

L'articolo presenta la biografia linguistica di una giovane studentessa dell'Università della Valle d'Aosta. Attraverso la storia di Cathy, che è nata e cresciuta in un ambiente multilingue, emergono le difficoltà che un plurilingue può incontrare per conservare il suo bagaglio linguistico ma anche i vantaggi che porta la padronanza di più codici.

The article features the linguistic biography of a young student at the Aosta Valley University. Thanks to Cathy's story, who was born and raised in a multilingual environment, one catches a glimpse of the difficulties a bi- or plurilingual person can experience in maintaining his/her linguistic acquisitions, as well as the advantages that come with mastering several languages.

\section{INDEX}

Keywords : linguistic biography, identity, weak languages, multilingualism Parole chiave : biografia linguistica, identità, lingue deboli, plurilinguismo

\section{AUTEUR}

\section{GABRIELLA VERNETTO}

Vallée d'Aoste (Italie) 